Notes

\title{
Quantitative Analysis of Methacycline Hydrochloride by Direct Potentiometry Using the Internal Solid Contact Sensor
}

\author{
Xian Xiang Sun ${ }^{* \dagger}$ and Hassan Y. ABoul-EnEIN** \\ *Department of Chemical Engineering, Jiangsu Polytechnic University, Changzhou 213016, P. R. China \\ **Pharmaceutical and Medicinal Chemistry Department, Pharmaceutical and Drug Industries Research Division, \\ National Research Center (NCR), Dokki, Cairo 12311, Egypt
}

\begin{abstract}
An internal solid contact sensor (ISCS) for the determination of methacycline hydrochloride (MC.Cl), Pt/PPy/PVC(MCPT), is described, based on the use of conducting poly(pyrrole) (PPy) as solid contact material and MC-phosphotungstate (PT) as the ion exchanger and dibutyl phthalate (DBP) as the plasticizer. A direct potentiometric method for the quantitative analysis of $\mathrm{MC} \cdot \mathrm{Cl}$ is also described. Under the condition of $\mathrm{pH} 2.7$, the linear concentration range, slope $\left(25^{\circ} \mathrm{C}\right)$ and detection limit of the sensor are $6.4 \times 1.0^{-6}-3.0 \times 1.0^{-3} \mathrm{M}, 52.4 \pm 0.2 \mathrm{mV} / \mathrm{decade}$ and $4.4 \times 1.0^{-6} \mathrm{M}$, respectively. The response time is $<5 \mathrm{~s}$. The determinations of $\mathrm{MC} \cdot \mathrm{Cl}$ in tablets were carried out by direct potentiometry. The average recovery and relative standard deviation are 100.1 and $0.7 \%(n=4)$, respectively.
\end{abstract}

(Received February 6, 2006; Accepted August 9, 2006; Published February 10, 2007)

The development of pharmaceutical, clinic and environmental analyses has led to the need of chemical sensors which are robust and easy to construct and to miniaturize, and which require no maintenance during the life period. Among those sensors, internal solid contact sensors (ISCSs), i.e. so-called all-solid state ion-selective electrodes based on the conducting polymers (e.g. PPy, poly(anline) (PAn) etc.) as ion-to-electron transducers have been given special attention, due to their good potential prospects when utilized in miniaturized separation/analytical or microfluidic systems such as HPLC, FIA, CE and $\mu$ TAC. ${ }^{1}$

Methacycline hydrochloride (MC.Cl), 4-(dimethylamino)1,4,4a,5,5a,6,11,12a-octahydro-3,5,10,12,12a-pentahydroxy-6methylene-1,11-dioxo-2-naphthacenecarboxamide monohydrochloride (Scheme 1), is one of tetracycline antibiotics family. These are broad-spectrum antibiotics including chlortetracycline (CTC), doxycycline (DC), methacycline (MC), minocycline (MNC) and oxytetracycline (OTC).

Tetracyclines are often used in veterinary medicine to prevent diseases, as an additive in animal feeds, ${ }^{2}$ and also as a genetic switch in gene expression, gene therapy and cancer therapy.,4 Therefore, searches for new analytical methods or techniques for the determination of tetracyclines in various samples have attracted a great deal of attentions. ${ }^{2,5,6}$

For the determination of $\mathrm{MC} \cdot \mathrm{Cl}$, some pharmacopoeias recommend high performance liquid chromatography (HPLC) $)^{7,8}$ and antibiotics-microbial assay. ${ }^{7}$ A few analytical methods including HPLC, spectrophotometry and phosphorimetry for the determination of $\mathrm{MC} \cdot \mathrm{Cl}$ in pharmaceutical analysis have been reported in the literature. To the best of our knowledge, no ISCS for determination of $\mathrm{MC} \cdot \mathrm{Cl}$ has been reported.

The comprehensive quality index (CQI) of a potentiometric electrode has recently been presented to evaluate quantitatively the performance of a electrode ${ }^{9}$ and to optimize the electropolymerization parameters for the preparation of PPy or Pan. ${ }^{5}$

$\dagger$ To whom correspondence should be addressed.

E-mail: sunxxiang@sohu.com
A method for calculating the value of CQI is based on the procedure that each of the four experimental factors: slope $(S)$, linear concentration range (LR), limit of detection (LD) and response time $(T)$, of a electrode is quantitated to be one number (less than 1.2), according to a given formula and a weighting factor. This was documented to be efficient. ${ }^{9}$ In the present paper, development of the sensor, Pt/PPy/PVC(MC-PT) for determination of $\mathrm{MC} \cdot \mathrm{Cl}$ is described, on the basis of optimizing the electropolymerization conditions including monomer (pyrrole) concentration, and concentration of supporting electrolyte and selecting of the electroactive substance in composition of PVC sensing membrane by the magnitude of CQI value.

\section{Experimental}

\section{Reagents and materials}

Phosphotungstic acid, sodium tetraphenylborate, and silicotungstic acid were all analytical reagent grade and purchased from Fluka. Dioctyl phthalate (DOP), dibutyl phthalate (DBP), tetrahydrofuran (A.R) and poly(vinyl chloride) (PVC) were obtained from commercial sources. Methacycline hydrochloride $(\mathrm{MC} \cdot \mathrm{Cl})$ is of Chinese pharmacopoeia grade,${ }^{8}$ and was obtained from local pharmaceutical chemical manufacturers. All the reagents and solvents are of analytical grade. Deionized water is used throughout the study. Diluted hydrochloride acid $(\mathrm{pH}$ 2.7 ) is prepared for use as a background solution for preparation<smiles>C=C1c2cccc(O)c2C(=O)C2=C(O)C3(O)C(=O)C(C(N)=O)=C(O)C(=O)C3C(O)C12</smiles>

Scheme 1 Structure of methacycline hydrochloride. 


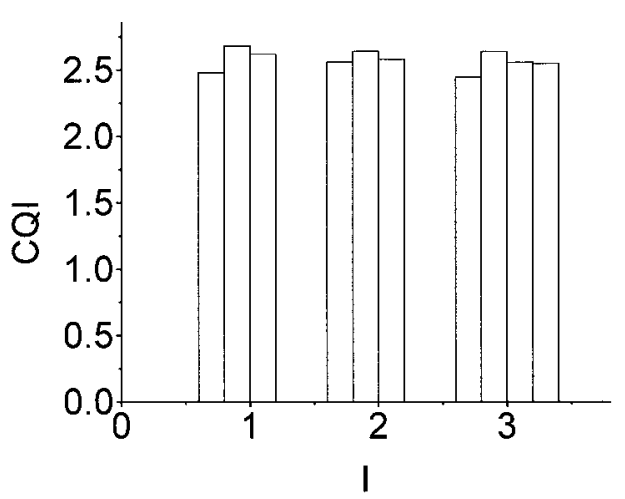

Fig. 1 Optimization of electrochemical preparation of PPy (Pt substrate electrode): 1 , concentration of pyrrole, $0.10,0.25$ and 0.30 $\mathrm{M} ; 2$, concentration of $\mathrm{KCl}, 0.50,1.00$ and $1.50 \mathrm{M} ; 3$, repeating scan times, 10, 15, 20 and 30.

of $\mathrm{MC} \cdot \mathrm{Cl}$ standard solution by sequential dilution. Platinum $(\mathrm{Pt})$ or glassy carbon $(\mathrm{GC})$ disk electrodes are a product of BAS Co. (USA). The saturated calomel electrode (SCE) is used as a reference electrode throughout the study.

\section{Preparation of sensor}

The preparation of each of three ion-pair compounds including MC-PT, MC-TPB and MC-SiT, and of the corresponding PVC sensing solutions are conducted with the protocol described elsewhere. ${ }^{10}$ For the preparation of PVC sensing solution with MC-PT as the electroactive substance, in short, about $65 \mathrm{~mL}$ of $0.01 \mathrm{M}$ solution of $\mathrm{MC} \cdot \mathrm{Cl}$ is mixed with $20 \mathrm{~mL}$ of $0.01 \mathrm{M}$ solution of phosphotungstic acid under stirring. The resulting yellow precipitate is filtered off, washed with water, and dried at $60^{\circ} \mathrm{C}$. The corresponding PVC sensing solution is then prepared by dissolving $10.0 \mathrm{mg}$ of MC-PT, 45.0 $\mathrm{mg}$ of DBP and $45.0 \mathrm{mg}$ of PVC in $3 \mathrm{~mL}$ of tetrahydrofuran.

The procedures of pretreating a substrate electrode (Pt or GC) surface and of modifying PPy or Pan film on substrate in a three electrode-cell, i.e. disc electrode used as the working electrode, SCE as reference electrode and platinum wire electrode as counter electrode, by repeating potential scan method, and of preparation of ISCS by dipping-coating procedures are the same as described by Sun et al. ${ }^{5,6}$ The following conditions are used for preparation of PPy or Pan film on substrate for a sensor. Preparation of PPy film: $1.0 \mathrm{M}$ of $\mathrm{KCl}$ contained $0.25 \mathrm{M}$ pyrrole, cycle potential scan 15 (or 20) times for Pt (or GC) over the potential range of -0.1 to $+0.7 \mathrm{~V}$, scan rate $80 \mathrm{mV} / \mathrm{s}$.

Preparation of Pan film: $1.0 \mathrm{M}$ of $\mathrm{HCl}$ contained $1.0 \mathrm{M}$ aniline, cycle scan 15 (or 20) times for Pt (or GC) in the potential range of -0.1 to $+0.8 \mathrm{~V}$, scan rate $60 \mathrm{mV} / \mathrm{s}$. The prepared sensor is conditioned by soaking into $1.0 \times 10^{-3} \mathrm{M}$ of $\mathrm{MC} \cdot \mathrm{Cl}$ solution for $2 \mathrm{~h}$ before it was used. When not in use, the sensor is stored in air. For simplicity, the following notations are used to refer the names of a sensor or ISE: substrate/solid contact/PVC (ion-pair), e.g. Pt/PPy/PVC(MC-PT), PVC (ionpair), e.g. $\mathrm{PVC}(\mathrm{MC}-\mathrm{TPB}$ ). The precision $\mathrm{pH}$-meter (Model pHs-2) instrument is used for the measurement of electromotive force (emf). A sensor and SCE are used as the indicating electrode and the reference electrode, respectively. The measurement of emf is carried out at room temperature in the following cell: SCE//test solution/ISE. The test solutions are constantly stirred with magnetic stirrers.

Evaluation of the comprehensive quality of sensor ${ }^{5,9}$

The value of CQI for a given sensor is caculated using the

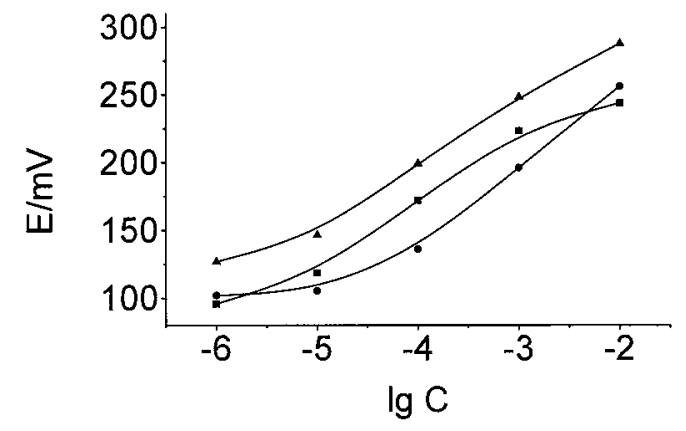

Fig. 2 The response characteristics of three sensors: $\mathbf{}$, sensor 1 (Pt/Ppy/PVC(MC-PT)); $\boldsymbol{\bullet}$, sensor 2 (Pt/PAn/PVC(MC-TPB)); $\boldsymbol{\Delta}$, sensor 3 (Pt/Ppy/PVC(MC-SiT)).

following equation: $\mathrm{CQI}=\Sigma f_{\mathrm{i}} P_{\mathrm{i}}$, where $f_{\mathrm{i}}$ denotes a weighting factor whose values are 0.38 for $f_{1}, 0.30$ for $f_{2}, 0.22$ for $f_{3}$ and 0.10 for $f_{4}$, respectively, and $P_{\mathrm{i}}$ denotes the devoting of one of performances, which include $S$ (mV/decade), LR (M), LD (M) and $T$ (s) which is defined as the time needed for the sensor to reach a stable potential value after a concentration step from $10^{-4}$ to $10^{-3} \mathrm{M}$ of the primary ion, ${ }^{11}$ to the value of CQI, and the values can be calculated from the following formula: $P_{1}=S / 20$, $P_{2}=\lg [\mathrm{LR}]_{\text {upper limit }}-\lg [\mathrm{LR}]_{\text {lower limit }}, P_{3}=a \mathrm{LD}_{\max } / \mathrm{LD}_{\mathrm{i}}, P_{4}=b / T_{\mathrm{i}}$. The " $a$ " and " $b$ " used in present work are 1.5 and 30 , respectively. ${ }^{5,9}$ The largest CQI value gives the highest quality of the sensor, indicating the best polymerization parameter as well.

\section{Results and Discussion}

\section{Optimization of preparation of PPy film}

Previous experimental investigations ${ }^{5,6}$ have found that the conditions/parameters of electropolymerization for pyrrole (or aniline) could affect the response performances of ISCSs to some extent. Our preliminary experiment indicates that selection of anionic sort of electrolyte, e.g. $\mathrm{Cl}^{-}, \mathrm{NO}_{3}{ }^{-}$and $\mathrm{SCN}^{-}$, as one of these conditions, could also influence the response behavior of ISCS, probably due to the different sizes and lipophilities. The effects of pyrrole concentration, from 0.10 to $0.30 \mathrm{M}$, of the sort of electrolyte (e.g., $\mathrm{KCl}, \mathrm{KNO}_{3}, \mathrm{KSCN}$ ), of the concentration of $\mathrm{KCl}$, from 0.5 to $1.5 \mathrm{M}$, and of the repeated scan times, from 10 to 30 times, on the potential response of ISCS are experimentally examined by using Pt substrate electrode. These parameters are optimized by using the CQI values. The ISCSs with $\mathrm{Cl}^{-}$ions as doping under the condition of $0.25 \mathrm{M}$ pyrrole had the largest values of CQI (>2.6). Part of the results obtained shown in Fig. 1, so the following electropolymerization parameters of pyrrole are the best selected: $0.25 \mathrm{M}$ pyrrole, $1 \mathrm{M} \mathrm{KCl}$, repeating scan times 15 (or 20 times for GC) and scan potential range from -0.1 to $+0.7 \mathrm{~V}$. It is of interest that the electropolymerization conditions are consistent with the optimized conditions of the preparation of PPy for the two sensors Pt/Ppy/PVC(OTC-PT) $)^{5}$ and Pt/Ppy/PVC(DC-TPB). ${ }^{6}$ For the preparation of PAn film, the optimized conditions of electropolymerization for the preparation of Pt/PAn/PVC(DC-TPB $)^{6}$ are directly applied as follows: $1.0 \mathrm{M}$ aniline, $1.0 \mathrm{M} \mathrm{HCl}$, repeated scan times 15 (or 20) times for $\mathrm{Pt}$ (or GC) and scan potential range from -0.1 to $+0.8 \mathrm{~V}$.

\section{Response characteristics of sensor}

The response characteristics of the sensors prepared with three ion-pair compounds (MC-PT, MC-TPB and MC-SiT) in PPy- 


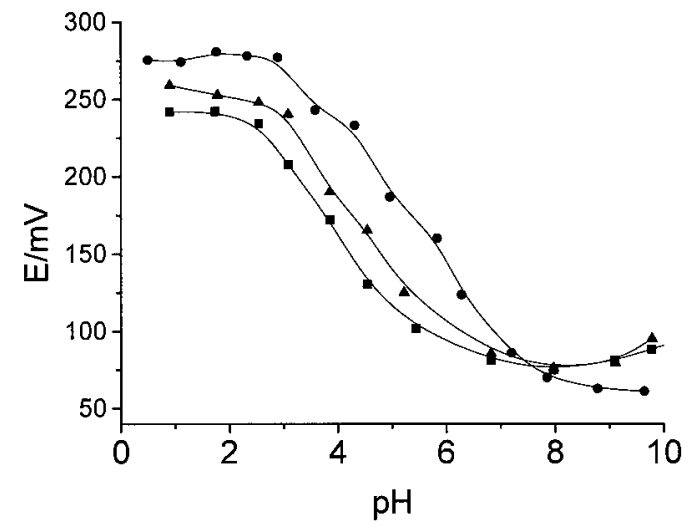

Fig. 3 The effects of $\mathrm{pH}$ on the responses of the sensors: $\mathbf{\square}$, sensor 1 (Pt/Ppy/PVC(MC-PT));, , sensor 2 (Pt/PAn/PVC(MC-TPB)); $\boldsymbol{\Delta}$, sensor 3 (Pt/Ppy/PVC(MC-SiT)).

based system and Pan-based system are compared by the magnitude of CQI. The values of CQI obtained (data not given) show that the sensors based on one of three ion-pair compounds in PPy-based system exhibit better response characteristics than sensors prepared with Pan-based system. Figure 2 indicates only the potential response of the ISCSs based on Pt substrate For two of the ISCSs based on GC substrate, the response slope $(\mathrm{mV} /$ decade), linear concentration range $(\mathrm{M})$ and detection limit (M) of these sensors are given as follows: 55.3, $6.5 \times 10^{-6}-7.8$ $\times 10^{-3}, 3.8 \times 10^{-6}$ for GC/PPy/(MC-PT); $50.8,4.8 \times 10^{-6}-8.2 \times$ $10^{-3}, 2.6 \times 10^{-6}$ for $\mathrm{GC} / \mathrm{PPy} /(\mathrm{MC}-\mathrm{TPB})$. The responses of all sensors are very rapid, and the response times are $<10 \mathrm{~s}$. The sensor, $\mathrm{Pt} / \mathrm{PPy} /(\mathrm{MC}-\mathrm{PT})$ is further used in the following experiments. The repeatability of the sensors is investigated by replicate emf measurements in series of $\mathrm{MC} \cdot \mathrm{Cl}$ standard solutions, resulting in a standard deviation of slopes of 0.24 $\mathrm{mV} /$ decade $(n=4)$. The potential drifts are used to evaluate the stability of the sensor, i.e. emf values at $1.0 \times 10^{-3} \mathrm{M}$ of $\mathrm{MC} \cdot \mathrm{Cl}$ solution are monitored continuously for $6 \mathrm{~h}$. The potential drifts obtained are $\leqslant 0.15 \mathrm{mV} / \mathrm{h}$.

The effect of $\mathrm{pH}$ on the response of the sensor is examined by measuring the potential variation in the emf at $1.0 \times 10^{-3} \mathrm{M}$ of $\mathrm{MC} \cdot \mathrm{Cl}$ solution. The results are shown in Fig. 3, we can see that only small changes of potential occurred in the $\mathrm{pH}$ ranges over $1.5-3.0$ and $7.0-9.0$, while the potentials vary sharply with the $\mathrm{pH}$ at $3.0-7.0$. It is known that $\mathrm{MC} \cdot \mathrm{Cl}$ solutions are unstable at basic or at acidic conditions $(e . g . \mathrm{pH}<2) . \quad \mathrm{A} \mathrm{pH}$ range of $2.0-3.0$ can be selected as the $\mathrm{pH}$ working range for the sensor. To eliminate the effects of $\mathrm{pH}$, diluted hydrochloric acid with a fixed $\mathrm{pH}$ value ( $\mathrm{pH} 2.7$ used in this study) is used as the background solution to prepare the $\mathrm{MC} \cdot \mathrm{Cl}$ standard solutions tested.

The selective coefficients $\left(K_{\mathrm{i}, \mathrm{j}}^{\mathrm{pot}}\right)$ of the sensor are investigated by separate solution methods $\left(a_{\mathrm{i}}=a_{\mathrm{j}}=10^{-3} \mathrm{M}\right)$ and results are summarized in Table 1. As can be seen from Table 1, inorganic cations $\left(\mathrm{K}^{+}, \mathrm{Na}^{+}, \mathrm{Ca}^{2+}, \mathrm{Mg}^{2+}, \mathrm{Al}^{3+}\right)$, organic compounds (e.g. citric acid and L-glutamic acid, D,L-alanine), and MNC, OTC do not interfere with the potential measurement, except that DC interferes slightly. Moreover, the excipients, such as corn starch, gelatin, and magnesium stearate found in the conventional formulations, do not influence the measurement. Moreover, MNC and OTC do not interfere with the potential measurement when one of the sensors (Pt/Pan/PVC(MC-TPB), GC/PPy/PVC(MC-TPB), GC/PPy/PVC(MC-PT) and Pt/PPy/ $\mathrm{PVC}(\mathrm{MC}-\mathrm{SiT})$ ) is used as indicating electrode. Table 1 gives also the values of $K_{i, j}^{\text {pot }}$ for electrode PVC(MC-PT) for the sake
Table 1 Selective coefficients of the sensor

\begin{tabular}{|c|c|c|}
\hline \multirow{2}{*}{ Interfering species (j) } & \multicolumn{2}{|c|}{$\log K_{\mathrm{MC}, \mathrm{J}}^{\mathrm{pot}}$} \\
\hline & Pt/PPy/PVC(MC-PT) & PVC(MC-PT) \\
\hline $\mathrm{Na}^{+}$ & -1.71 & -1.12 \\
\hline $\mathrm{K}^{+}$ & -1.29 & -1.32 \\
\hline $\mathrm{NH}_{4}^{+}$ & -2.09 & -1.49 \\
\hline $\mathrm{Ca}^{2+}$ & -3.11 & -2.60 \\
\hline $\mathrm{Mg}^{2+}$ & -2.90 & -2.20 \\
\hline $\mathrm{Al}^{3+}$ & -3.56 & -2.72 \\
\hline Citric acid & -1.10 & -1.20 \\
\hline L-Glutamic acid & -0.28 & -1.02 \\
\hline Minocycline·HCL & -0.44 & -0.84 \\
\hline Oxytetracycline $\cdot \mathrm{HCl}$ & -1.26 & -1.25 \\
\hline Doxycycline $\cdot \mathrm{HCl}$ & 0.27 & 0.01 \\
\hline
\end{tabular}

of comparison. It can also be seen that the selectivity of the sensor toward the inorganic cations is slightly improved in comparison with ISE.

\section{Analytical application of the sensor}

The direct potential method is used to assess the reliability of the sensor by determining $95.8-383.2 \mu \mathrm{g} / \mathrm{mL}$ of $\mathrm{MC} \cdot \mathrm{Cl}$. The recovery range and standard deviation are $99.9-100.2 \%, \pm 0.5-$ $\pm 0.8 \%(n=4)$, respectively. The determination of $\mathrm{MC} \cdot \mathrm{Cl}$ in pharmaceutical tablets ( $c a .100 \mathrm{mg} \mathrm{MC} \cdot \mathrm{Cl} /$ tablet, local pharmaceutical works, batch number: 20000113B) is carried out by means of the direct potentiometry and using the same protocols for sample analysis ${ }^{10}$ with the sensor. Results are compared with those of the pharmacopoeia method (HPLC). ${ }^{8}$ The mean value and relative standard deviation obtained are $96.1 \mathrm{mg} /$ tablet and $0.65 \%(n=6)$ respectively, while the mean value obtained with the pharmacopoeia method is 96.4 $\mathrm{mg} / \mathrm{tablet}$.

A novel sensor, Pt/PPy/PVC(MC-PT), sensing $\mathrm{MC} \cdot \mathrm{Cl}$ has been developed on the basis of selection of electroactive substances in the composition of the PVC sensing membrane and optimization of electropolymerization parameters of pyrrole. The sensor responses quickly and exhibits better selectivity. The direct potentiometric method for quantitative analysis of $\mathrm{MC} \cdot \mathrm{Cl}$ with the sensor has good accuracy and a large dynamic range. The sensor was successfully applied to the determination of $\mathrm{MC} \cdot \mathrm{Cl}$ in tablets.

\section{References}

1. L. J. Nagels and I. Poels, Trends Anal. Chem., 2000, 19(7), 410.

2. W. A. Moats, J. Agric. Food Chem., 2000, 48, 2244.

3. J. S. Yu, E. M. Sena, and W. Paulus, Cancer Res., 1996, 56,5423 .

4. M. Marble, Immunotherapy Weekly, 1999, 7(26), 10.

5. X. X. Sun, X. Zhang, and H. Y. Aboul-Enein, IL. FARMACO, 2004, 59, 307.

6. X. X. Sun and H. Y. Aboul-Enein, Talanta, 2002, 58, 387.

7. U. S. Pharmacopoeia, XXIII, 1995, The United States Phamacopoeia Convetion Inc., Rockville, MD.

8. Chinese Pharmacopoeia, Part II, 2000, Commission of the Ministry of Health Beijing.

9. X. X. Sun, Fenxi Ceshi Xuebao, 2003, 4, 1.

10. H. Y. Aboul-Enein, X. X. Sun, and C. J. Sun, Sensors, 2002, 2, 424.

11. R. P. Buck and E. Lindner, Pure Appl. Chem., 1994, 66(12), 2527. 